\title{
Exploring the Power Flow Solution Space Boundary
}

\author{
Ian A. Hiskens, Senior Member and Robert J. Davy
}

\begin{abstract}
A knowledge of the structure of the boundary of solutions of the power flow problem is important when analyzing the robustness of operating points. This paper proposes a predictor-corrector technique to assist in exploring that structure. Points on the solution boundary satisfy the power flow equations together with an equation which forces the power flow Jacobian to be singular. Curves of such points result from freeing two parameters of the system. The proposed technique follows those curves. A simple example is used to illustrate the complex nature of the power flow solution space.
\end{abstract}

Index Terms-Continuation methods, Jacobian singularity, maximum loadability, power flow, solution space boundary.

\section{INTRODUCTION}

$\mathbf{P}$ OWER system operation is constrained by loadability limits that closely match the boundedness of the power flow solution space [1]. Operation near the solution space boundary often results in undesirable system behavior that can be associated with reduced stability margins. ${ }^{1}$ Quantifying the boundary is therefore an important aspect of assessing system security.

In an electricity market environment, loadability restrictions can cause congestion, thereby influencing energy trading arrangements and distorting locational energy prices. A clearer understanding of the restrictions placed by power flow boundedness would therefore assist operators and traders in their decision-making, with a consequent improvement in power system security and price stability.

Unfortunately, the structure of the solution space boundary has not been clearly established. Indications are that it can display quite complicated behavior [3]. An hypothesis in [4] suggested that the solution space is convex, though a counterexample is provided later in our paper. (The solution space shown in Fig. 10 has a hole through it.)

Techniques for exploring the power flow solution space boundary therefore have an important role to play in the analysis of power systems. This paper describes a continuation method that generates nomograms of the solution space boundary.

Manuscript received September 8, 2000. This work was supported by the Australian Research Council through the project grant "Analysis and Assessment of Voltage Collapse," the EPRI/DoD Complex Interactive Networks/Systems Initiative, and the Grainger Foundation.

I. A. Hiskens is with the Department of Electrical and Computer Engineering, University of Illinois at Urbana-Champaign, Urbana, IL, USA.

R. J. Davy is with CSIRO Land and Water, Canberra, Australia.

Publisher Item Identifier S 0885-8950(01)06061-8.

${ }^{1}$ As an operating point moves closer to the solution boundary, so to does a corresponding unstable low-voltage solution. The stability boundary is tied to this low-voltage solution. Therefore as the two solution points merge, the stability boundary approaches the operating point, and the stability region shrinks [2].

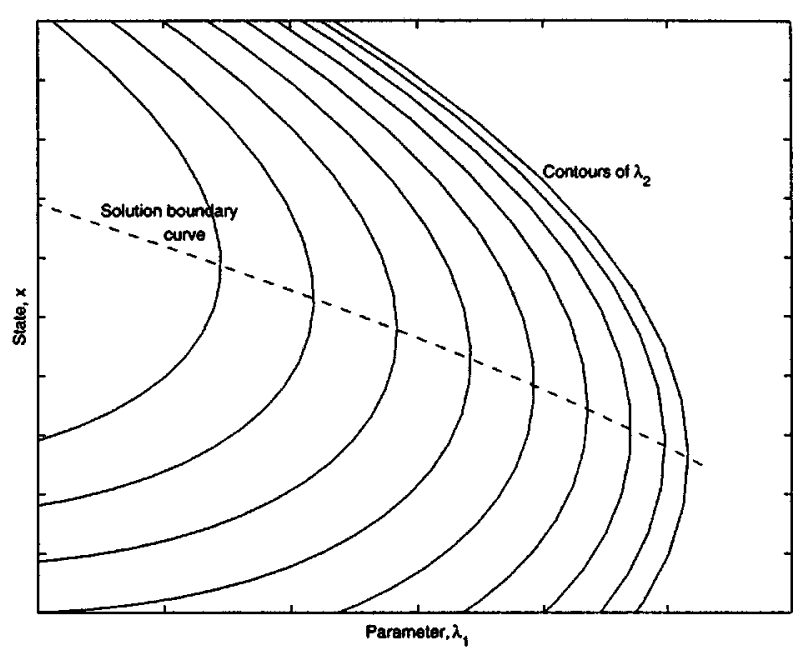

Fig. 1. Power flow solution curves.

The boundedness of power flow solutions is illustrated in Fig. 1. Each curve (or contour) is obtained by freeing a single parameter $\lambda_{1}$ and monitoring a state $x$. The family of curves is obtained by generating contours for a range of values of a second parameter $\lambda_{2}$. Notice that along each individual curve, $\lambda_{1}$ reaches a maximum value at a turning point. Those extreme points lie on the boundary of the solution space, and have been referred to as "points of maximum loadability." Fig. 1 also shows a dashed curve that joins the extreme points. That curve describes the solution space boundary in terms of parameters $\lambda_{1}$ and $\lambda_{2}$. It could be obtained by interpolating between the turning points of each contour. However a more systematic approach is described in this paper.

For a single free parameter, as illustrated by the individual curves of Fig. 1, numerous techniques are available for determining a point on the solution space boundary. They include direct methods [5]-[8], optimization methods [9], and continuation (homotopy) methods [10]-[15]. The continuation methods are of most interest in this paper, as they form the basis for generating curves that lie on the solution space boundary. A summary of one such method, the Euler homotopy approach, is provided in Section III.

A number of continuation techniques for determining solution boundary curves have been proposed [16], [17]. In this paper we describe the implementation of one of those techniques, and its use in exploring the power flow solution space boundary.

The paper is organized as follows. Section II provides an analytical description of solution boundary curves. An overview of continuation methods is provided in Section III. Section IV describes the implementation of a continuation method for finding 
solution boundary curves. A number of examples are given in Section V. The influence of reactive power limits is considered in Section VI, and conclusions are presented in Section VII.

\section{SOLUTION BOUNDARY CURVES}

The standard power flow problem can be expressed as the set of equations,

$$
f(x, \lambda)=0
$$

where $x \in \mathbb{R}^{n}, \lambda \in \mathbb{R}^{p}$ and $f: \mathbb{R}^{n+p} \rightarrow \mathbb{R}^{n}$. If a single parameter is free to vary, then $p=1$, and the problem becomes one of $n$ equations in $n+1$ unknowns, i.e., the system is underconstrained. Solutions are curves, not points. Each curve of Fig. 1 is an example. If $p=2$, i.e., two free parameters, then (1) defines a surface. The collection of curves in Fig. 1 provides contours of such a surface.

For a point to lie on the solution space boundary, it must firstly be a solution of the power flow, i.e., it must satisfy (1). However an additional requirement is that the power flow Jacobian must be singular, i.e.,

$$
\operatorname{det} f_{x}(x, \lambda)=0
$$

where $f_{x}$ refers to the Jacobian $\partial f / \partial x$. (We shall use this notation throughout the paper.) This places an extra constraint on boundary points.

Consider the case of a single free parameter, so that the number of unknowns is $n+1$. Boundary solutions must satisfy the $n$ equations of (1) together with the extra equation (2), a total of $n+1$ equations. Because the number of unknowns equals the number of equations, the solution boundary in this case is composed of points. Fig. 1 illustrates this. The solution boundary for each individual curve is given by an isolated turning point.

In general, if there are $p$ free parameters, then the system will have $n+p$ unknowns and $n+1$ equations. So the solution boundary in general is a $(p-1)$-manifold. Consider the surface, corresponding to $p=2$, described by the collection of curves in Fig. 1. It can be seen that the solution boundary is composed of the union of the boundary points of the contour curves. The boundary is the 1-manifold (dashed curve) shown in that figure.

In actually calculating boundary points, it is difficult to implement the constraint (2) directly in algorithms that solve sets of nonlinear simultaneous equations. However (2) can be effectively implemented as,

$$
\begin{aligned}
f_{x}(x, \lambda) y & =0 \\
y^{t} y & =1
\end{aligned}
$$

where $y \in \mathbb{R}^{n}$ is the right eigenvector corresponding to a zero eigenvalue of $f_{x}$. Because (3), (4) force $f_{x}$ to have a zero eigenvalue, they ensure that (2) is satisfied.

Points on the power flow solution space boundary are therefore described by

$$
\begin{aligned}
f(x, \lambda) & =0 \\
f_{x}(x, \lambda) y & =0
\end{aligned}
$$

$$
y^{t} y=1,
$$

a set of $2 n+1$ equations in $2 n+p$ unknowns. It again follows that when $p=2$, the set of equations (5)-(7) defines solution boundary curves.

\section{AN Overview OF A CONTINUATION METHOD}

As mentioned earlier, if a set of equations is underdetermined, with one more unknown than constraint, then solutions will be curves rather than points. We are interested in determining such curves. One method is to generate successive points along the curve using an Euler homotopy approach [10]. This is a predictor-corrector algorithm that was successfully applied to the power flow problem in [11]. In this section we review this approach to generating solution curves. The details of implementing the algorithm for generating boundary curves are provided in Section IV.

To describe the algorithm, it is convenient to write (5)-(7), with $p=2$, as a general set of equations,

$$
\phi(z)=0
$$

where

$$
z=\left[\begin{array}{l}
x \\
y \\
\lambda
\end{array}\right] \in \mathbb{R}^{\eta+1}, \quad \phi=\left[\begin{array}{c}
f \\
f_{x} y \\
y^{t} y-1
\end{array}\right]: \mathbb{R}^{\eta+1} \rightarrow \mathbb{R}^{\eta}
$$

and $\eta=2 n+1$. Assume we are at a point $z_{1}$ on the curve and wish to move to the next point.

The first step of the algorithm is the prediction of the next point on the curve. To do this, we find the vector that is tangent to the curve at $z_{1}$, and move along that vector a predefined distance $\tau$. This $\tau$ is a (scalar) control parameter that effectively determines the distance between successive points along the curve. In regions of high curvature, $\tau$ may need to be small. When the curve is almost linear, a large value of $\tau$ would suffice. The unit vector $v \in \mathbb{R}^{\eta+1}$ that is tangent to the curve (8) at $z_{1}$ is given by,

$$
\begin{aligned}
\left.\phi_{z}\right|_{z=z_{1}} v & =0 \\
\|v\| & =1
\end{aligned}
$$

where $\phi_{z}$ is the $\eta \times(\eta+1)$ Jacobian $\partial \phi / \partial z$. The prediction of the next point on the curve is,

$$
z_{p}=z_{1}+\tau v
$$

Having found the prediction point, we now need to correct to a point $z$ on the curve. The Euler method does this by solving for the point of intersection of the curve and a hyperplane that passes through $z_{p}$ and that is orthogonal to $v$. Points $z$ on this hyperplane are given by,

$$
\left(z-z_{p}\right)^{t} v=0
$$

or alternatively

$$
\left(z-z_{1}\right)^{t} v=\tau .
$$

Either (11) or (12) can be used. The point of intersection of the curve and the hyperplane is then given by,

$$
\phi(z)=0
$$




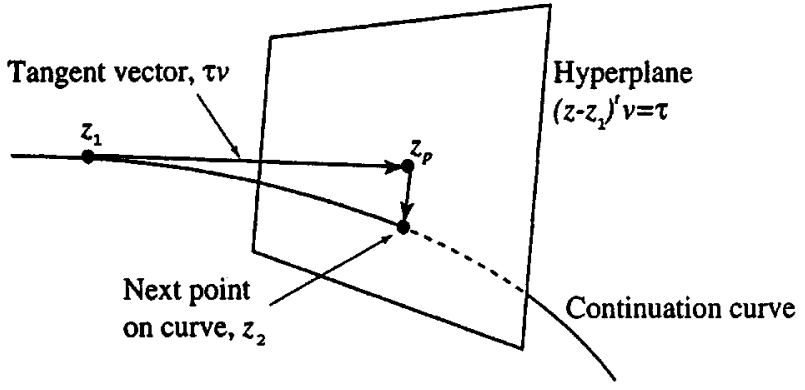

Fig. 2. Predictor-corrector process.

$$
\left(z-z_{1}\right)^{t} v=\tau
$$

Note that in (14), $z_{1}, v$ and $\tau$ are fixed, with $z$ being the only unknown. The first $\eta$ equations, which follow from (8), ensure the point is on the desired curve. The last equation, from (12), ensures the point is on the hyperplane. Together (13), (14) form a set of $\eta+1$ equations in $\eta+1$ unknowns. They can be solved using a standard technique such as Newton-Raphson. The predictor-corrector process is illustrated in Fig. 2.

After the second point $z_{2}$ on the curve has been determined, an approximate tangent vector can generally be used for obtaining successive points. The approximate tangent vector at the $i$ th point, which is used to calculate the $(i+1)$ th point, is given by,

$$
v_{i}=\frac{z_{i}-z_{i-1}}{\left\|z_{i}-z_{i-1}\right\|}
$$

Obtaining the approximate tangent vector involves much less computation than finding the exact tangent vector using (9), (10). However the approximation may not be adequate in regions of high curvature.

\section{IMPLEMENTATION}

In order to use a predictor-corrector technique to obtain a solution boundary curve, we need to find an initial point on that curve. Referring to Fig. 1, the simplest approach is to follow one of the power flow curves until a turning point is identified. That turning point provides an initial point on the solution boundary curve. Generating a boundary curve can therefore be viewed as a two stage algorithm: 1) find an initial boundary point, and 2) continue the boundary curve from that point. Over the first stage, only a single parameter $\lambda_{1}$ is free. Stage 2 requires the release of a second parameter $\lambda_{2}$.

During stage 1 , the tangent vector (generated as a by-product of the continuation process) can be used to identify a turning point. Referring to (9), the tangent vector along power flow curves, i.e., over stage 1 , can be written

$$
\left[\begin{array}{ll}
f_{x} & f_{\lambda}
\end{array}\right]\left[\begin{array}{l}
v_{x} \\
v_{\lambda}
\end{array}\right]=0 .
$$

Note that $v_{\lambda}$ is a scalar because there is only one free parameter at this stage. It can be seen from Fig. 1 that at turning points, the tangent to the power flow curve is vertical. As a curve is traversed through such a point, there is no change in the value of the parameter. This implies that at a turning point $v_{\lambda}=0$. By monitoring $v_{\lambda}$ along the power flow curve, a point that is close to a boundary point can be found.

The initial point of the boundary curve must satisfy (5)-(7). Recall that in finding this initial point, $\lambda_{1}$ is the only free parameter, with $\lambda_{2}$ still constrained at this stage. So (5)-(7) becomes a set of $2 n+1$ equations in $2 n+1$ unknowns, i.e., the initial point is uniquely defined. A technique such as Newton-Raphson can therefore by used to find the initial point. However such techniques rely on a good initial guess for the variables $x, y$ and $\lambda$. Estimates of $x$ and $\lambda$ are provided explicitly by the stage 1 continuation process. An estimate of $y$ is given by the tangent $v_{x}$. To see this, recall that at the boundary point $v_{\lambda}=0$. So from (15),

$$
f_{x}(x, \lambda) v_{x}=0
$$

From (6) it can be seen that $v_{x}$ provides an estimate of $y$.

Often more than one boundary point exists on the stage 1 power flow curve. Hence there are multiple potential starting points. This property is used when computing disconnected sections of the solution boundary, and is illustrated in the example of Section V-B.

During stage 2, along the boundary curve, the Jacobian of (5)-(7) has the form,

$$
J=\left[\begin{array}{ccc}
\frac{\partial f}{\partial x} & 0 & \frac{\partial f}{\partial \lambda} \\
\frac{\partial g}{\partial x} & \frac{\partial g}{\partial y} & \frac{\partial g}{\partial \lambda} \\
0 & \frac{\partial h}{\partial y} & 0
\end{array}\right]=\left[\begin{array}{ccc}
f_{x} & 0 & f_{\lambda} \\
\frac{\partial g}{\partial x} & f_{x} & \frac{\partial g}{\partial \lambda} \\
0 & 2 y^{t} & 0
\end{array}\right]
$$

where

$$
\begin{aligned}
& g=f_{x} y: \mathbb{R}^{2 n+2} \rightarrow \mathbb{R}^{n} \\
& h=y^{t} y-1 \\
& J \in \mathbb{R}^{(2 n+1) \times(2 n+2)} .
\end{aligned}
$$

When solving the corrector, (5)-(7) is augmented by an extra row corresponding to the equation describing the hyperplane, i.e., (11) or (12). This introduces an extra row into (16).

The $i$ th component of vector function $g$ is given by,

$$
g_{i}(x, y, \lambda)=\sum_{k=1}^{n} \frac{\partial f_{i}}{\partial x_{k}} y_{k}
$$

where $f_{i}$ is the $i$ th component of $f$, and $x_{k}$ and $y_{k}$ are the $k$ th components of $x$ and $y$ respectively. Therefore $\partial g / \partial x$ is an $(n \times$ $n$ ) matrix with $(i, j)$ th element,

$$
\left[\frac{\partial g}{\partial x}\right]_{(i, j)}=\sum_{k=1}^{n} \frac{\partial^{2} f_{i}}{\partial x_{k} \partial x_{j}} y_{k} .
$$

Similarly $\partial g / \partial \lambda$ is an $(n \times 2)$ matrix with elements,

$$
\left[\frac{\partial g}{\partial \lambda}\right]_{(i, j)}=\sum_{k=1}^{n} \frac{\partial^{2} f_{i}}{\partial x_{k} \partial \lambda_{j}} y_{k} .
$$

Most elements of $g_{x}$ and $g_{\lambda}$ are zero, resulting in an extremely sparse structure for $J$. Therefore the continuation process is tractable for large systems. 


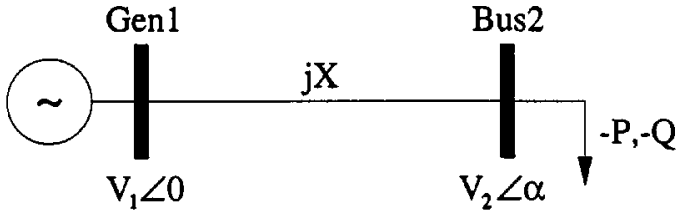

Fig. 3. Two bus system.

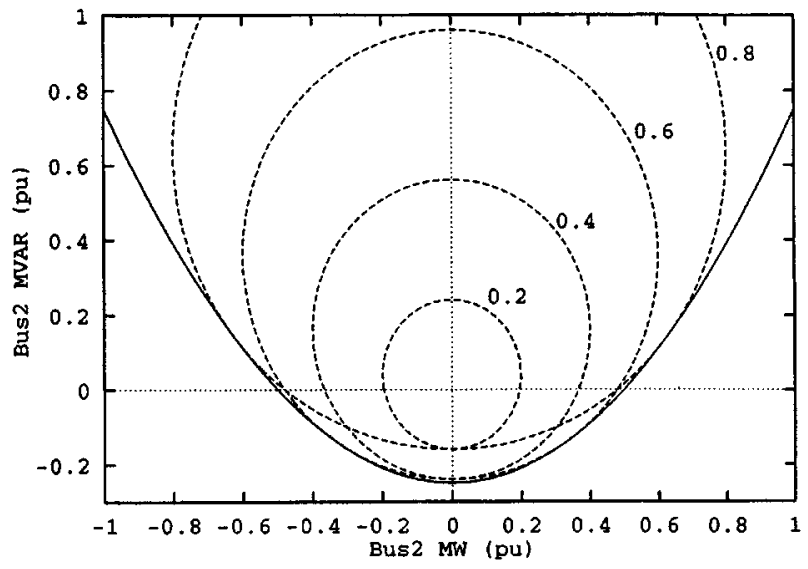

Fig. 4. Power circles and solution boundary curve. Contours of $V_{2}$.

\section{EXAMPLES}

\section{A. Two Bus System}

The numerical results obtained using the continuation algorithm described earlier may be verified analytically for a two bus system, such as shown in Fig. 3. In this system, Gen1 is a slack bus, Bus 2 is a PQ bus, and $V_{1}=X=1.0 \mathrm{pu}$.

Eliminating $\alpha$ from the real and reactive power balance equations for Bus 2 results in equations for power circles in the $P-Q$ plane,

$$
P^{2}+\left(\frac{V_{2}^{2}}{X}-Q\right)^{2}=\left(\frac{V_{1} V_{2}}{X}\right)^{2}
$$

These curves (circles) are shown in Fig. 4 as dashed lines. Each circle corresponds to a different value of $V_{2}$. There exists a boundary in the $P-Q$ plane beyond which there are no power flow solutions. At any point on that boundary, the power flow Jacobian $f_{x}$ is singular. It can easily be shown that points which lie on the boundary, i.e., that satisfy the power flow equations along with the requirement det $f_{x}=0$, are given by,

$$
Q=\frac{X}{V_{1}^{2}} P^{2}-\frac{V_{1}^{4}}{4 X} .
$$

Hence the solution boundary curve in the $P-Q$ plane is a parabola (remembering that $V_{1}$ and $X$ are fixed).

The solution boundary can be computed numerically by making the following observation. In $P-V_{2}$ space, with $Q$ held constant, boundary points occur when there is a change in the number of solutions as $P$ is varied. The dashed curves of Fig. 5 show solutions for various (fixed) values of $Q$. (These curves are analogous to Fig. 1. In this example $\lambda_{1}$ is $P$ and $\lambda_{2}$ is $Q$.) Using the continuation technique, and allowing $Q$ to be a free parameter, the boundary curve in $P-V_{2}$ space can be computed. It is shown in Fig. 5 as a solid curve. The same curve plotted

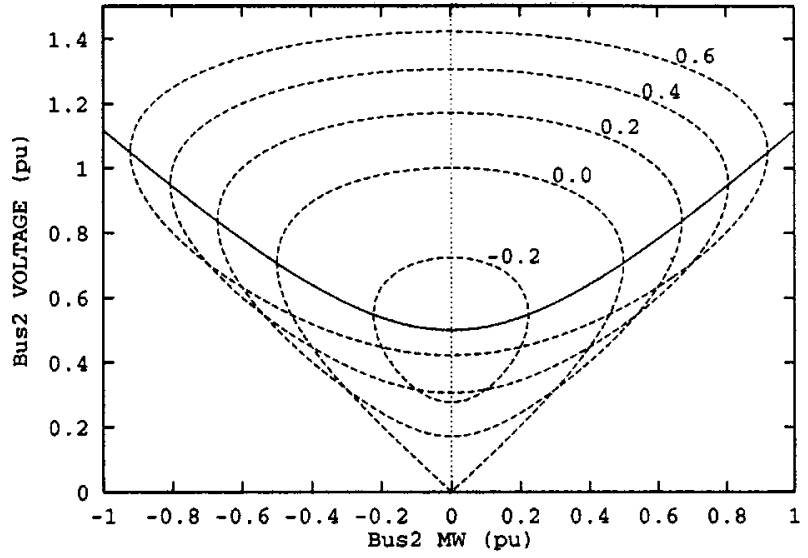

Fig. 5. $P V$ curves and solution boundary curve. Contours of $Q$.

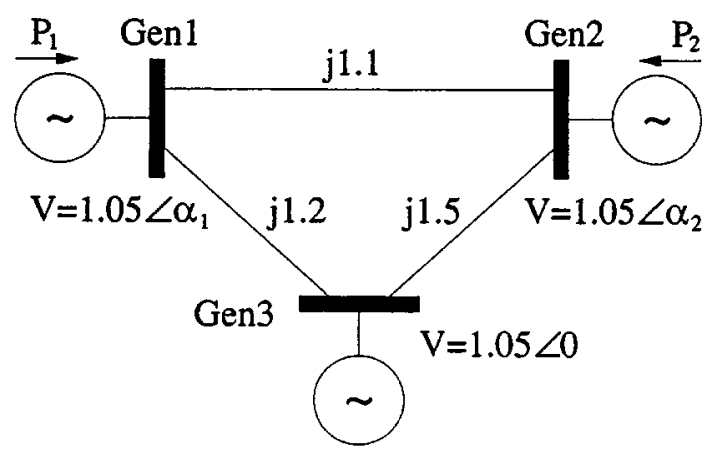

Fig. 6. Three bus system.

in $P-Q$ space is shown as a solid curve in Fig. 4. Note that it has the predicted parabolic form. Furthermore, it forms the boundary of the power circle diagrams and is tangential to the circles.

It is interesting to note that the contours (dashed lines) of Fig. 4 correspond to horizontal slices through Fig. 5, and the contours of Fig. 5 correspond to horizontal slices through Fig. 4. Together they provide a picture of the solution space in $P-Q-V_{2}$ space.

\section{B. Three Bus System}

This example explores the solution space boundary for the system of Fig. 6. Even though the system is small, it illustrates the complexity of the power flow solution space. The solution space boundary will be investigated for two cases. The first considers the boundary when $P_{1}$ and $P_{2}$ are free to vary, whilst the second presents nomograms of $P_{2}$ versus $Q_{2}$. The connection between these two cases will also be explored.

1) Case 1: $P_{2}$ versus $P_{1}$ : The power flow solution space projected onto the $P_{1}-P_{2}$ plane is shown in Fig. 7. In this figure, each curve corresponds to a distinct value of $\alpha_{1}$. The outer boundary of the solution space is clear. However there is also some folding within the solution space. The continuation technique can be used to locate all the boundary curves, including the inner folds.

Finding the boundary points amounts to finding those points where, if $P_{2}$ is held constant and $P_{1}$ is varied (or vice-versa), there is a change in the number of power flow solutions. Fig. 8 shows the power-angle curves at Gen 1 for various values of $P_{2}$. 


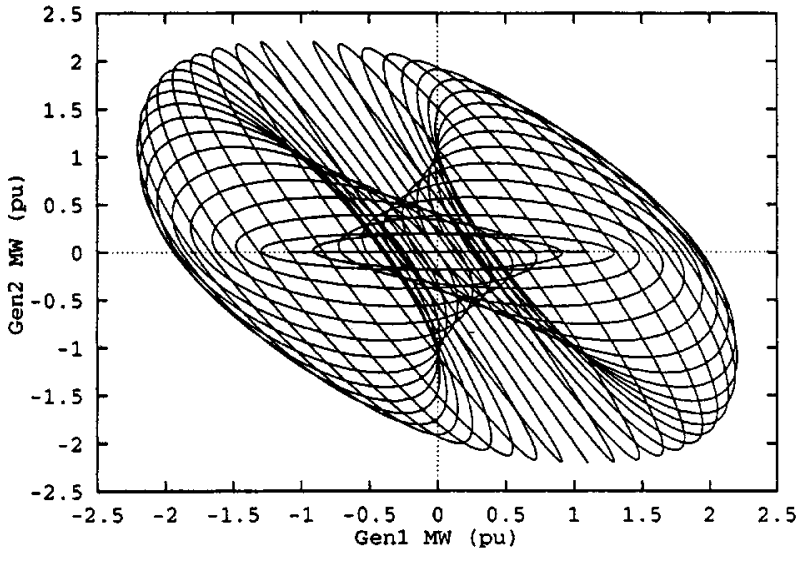

Fig. 7. Power flow solution space, $P_{1}-P_{2}$ view.

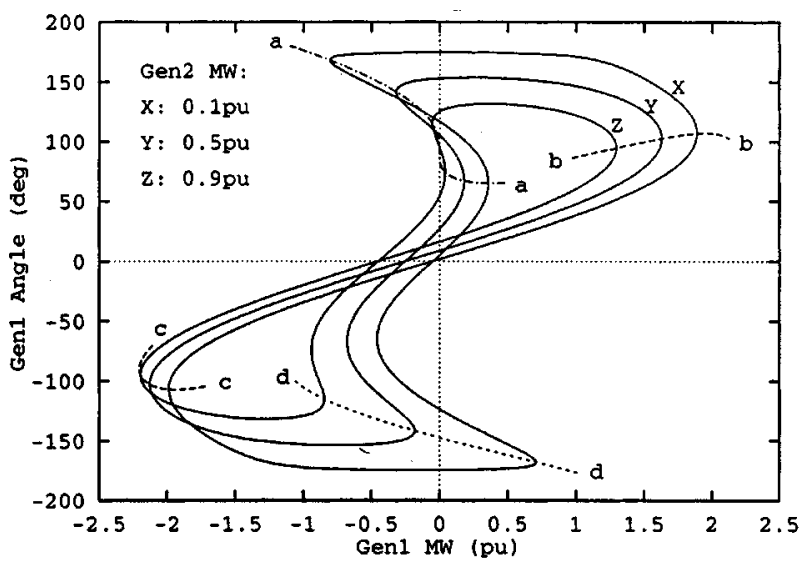

Fig. 8. Power-angle curves.

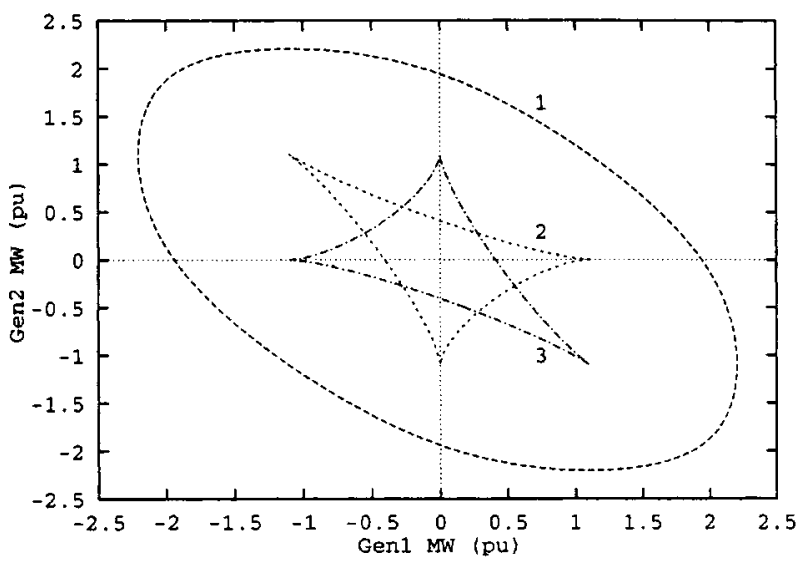

Fig. 9. Boundary curves in $P_{1}-P_{2}$ space.

(These curves are rotated by $90^{\circ}$ compared with the normal presentation of power-angle curves. They are oriented in this way to follow the bifurcation diagram convention of parameter on the horizontal axis and state on the vertical axis.) There exist several boundary (bifurcation) points with respect to $P_{1}$. Using any of these as a starting point, and releasing $P_{2}$, loci of boundary points may be computed (broken lines). Fig. 9 is obtained when these loci are plotted in $P_{1}-P_{2}$ space. Lines $c-c$ and $b-b$ in Fig. 8 form parts of curve 1 in Fig. 9. Likewise, line $d-d$ forms part of curve 2 , and line $a-a$ forms part of curve 3 . Curves 1, 2 and 3 are

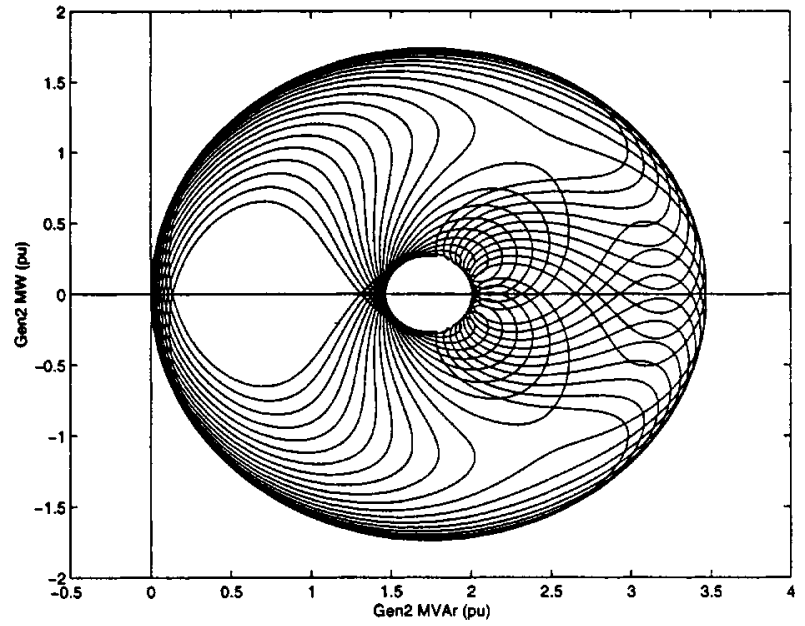

Fig. 10. Solution space, $Q_{2}-P_{2}$ view. Constant $P_{1}$ curves.

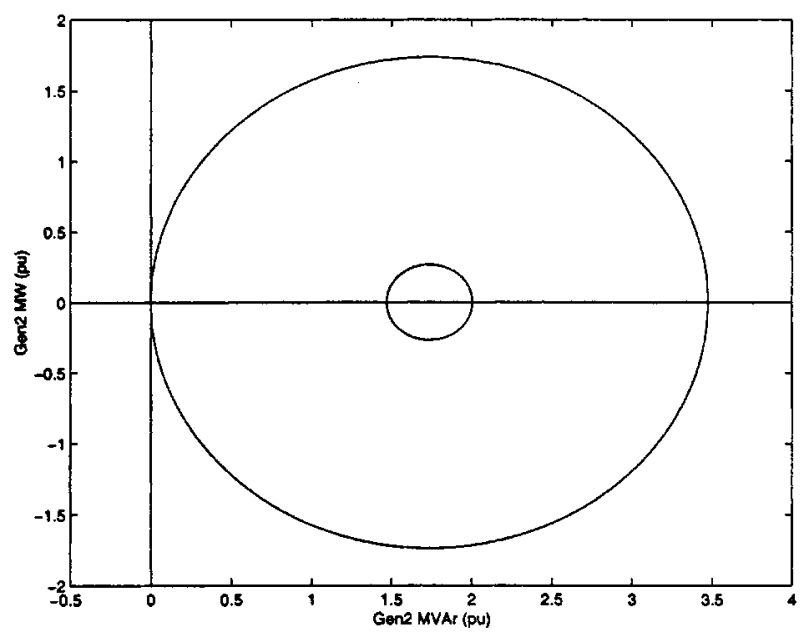

Fig. 11. Boundary curves in $Q_{2}-P_{2}$ space.

disconnected. Different starting points must be used to obtain the various curves. Curve 1 forms an outer boundary, beyond which no power flow solutions exist. Curves 2 and 3 correspond to the internal folds. They divide the solution space into regions where different numbers of equilibria exist [3].

2) Case 2: $P_{2}$ versus $Q_{2}$ : An interesting view of the solution space is obtained by plotting $P_{2}$ versus $Q_{2}$ for various values of $P_{1}$. The resulting curves are shown in Fig. 10. The solution space is clearly bounded. Of particular interest though is the hole through the middle of the solution space. Note also the convoluted form of the individual curves.

To obtain initial points on the outer and inner boundary curves, $Q_{2}$ was constrained to a value of $1.8 \mathrm{pu}$, corresponding to a slice through the hole. Parameters $P_{1}$ and $P_{2}$ were both allowed to vary. Initial boundary points were given by the bifurcation points of the resulting $P_{2}$ versus $\alpha_{2}$ curve. The boundary curves obtained from those initial points are shown in Fig. 11.

Even though it is not immediately apparent, Fig. 10 is closely related to power circles similar to those of Fig. 4. This becomes clearer in Fig. 12, where curves of $P_{2}$ versus $Q_{2}$ are plotted for various values of $\alpha_{2}$. Each curve has a circular shape. The 


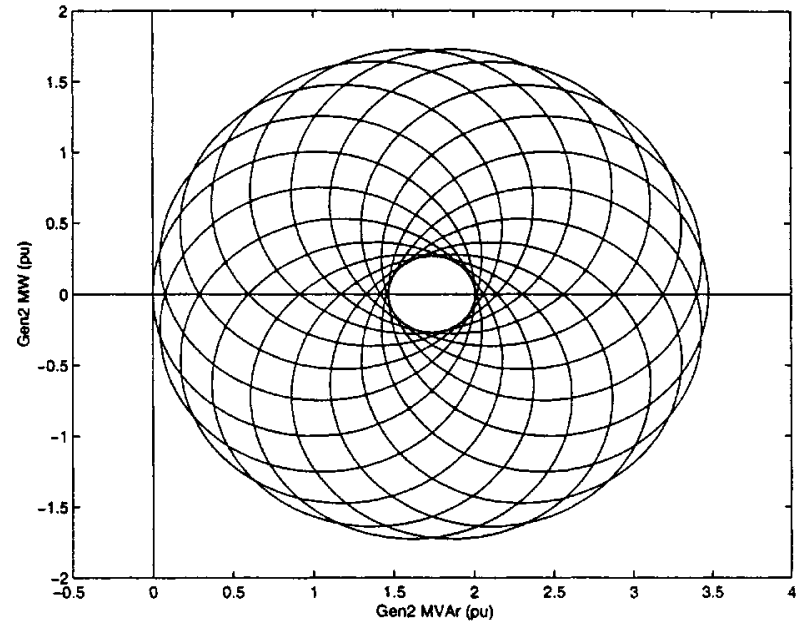

Fig. 12. Solution space, $Q_{2}-P_{2}$ view. Constant $\alpha_{2}$ curves.

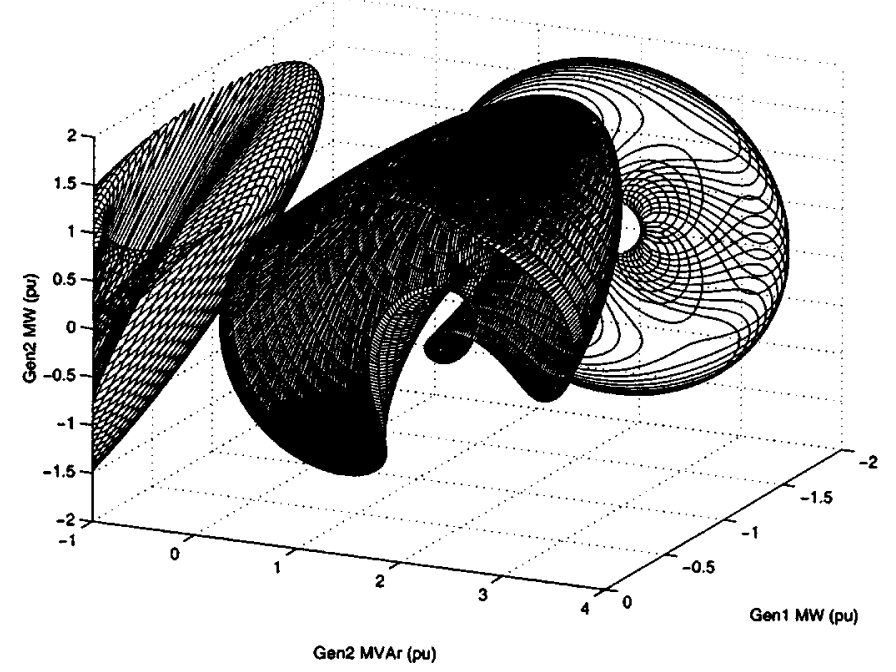

Fig. 13. Solution space, $P_{1}-Q_{2}-P_{2}$ view.

collection of curves all lie within the solution space boundaries identified in Fig. 11.

3) Connections: The projections of the power flow solution space given by Figs. 7 and 10 can be combined to show the solution space in the three dimensional $P_{1}-Q_{2}-P_{2}$ space. Fig. 13 shows half of this surface, together with its $P_{1}-P_{2}$ and $Q_{2}-P_{2}$ projections. (Half the surface was removed to reveal the inner folds.) The complicated features of this surface, including the folds and the hole, are now easier to comprehend.

\section{REACTIVE POWER LIMITS}

Reactive power limits of generators and SVCs can play a role in limiting the power flow solution space [18]. In such cases, the solution space boundary is composed of sections where the power flow Jacobian is singular, and other sections where a reactive power limit is enforced. Fig. 14 illustrates such a case. This is the same example as shown in Fig. 1, but now reactive power limits are considered. As in Fig. 1, the dashed line shows the boundary due to Jacobian singularity. However this is now truncated by the dotted line which corresponds to points where

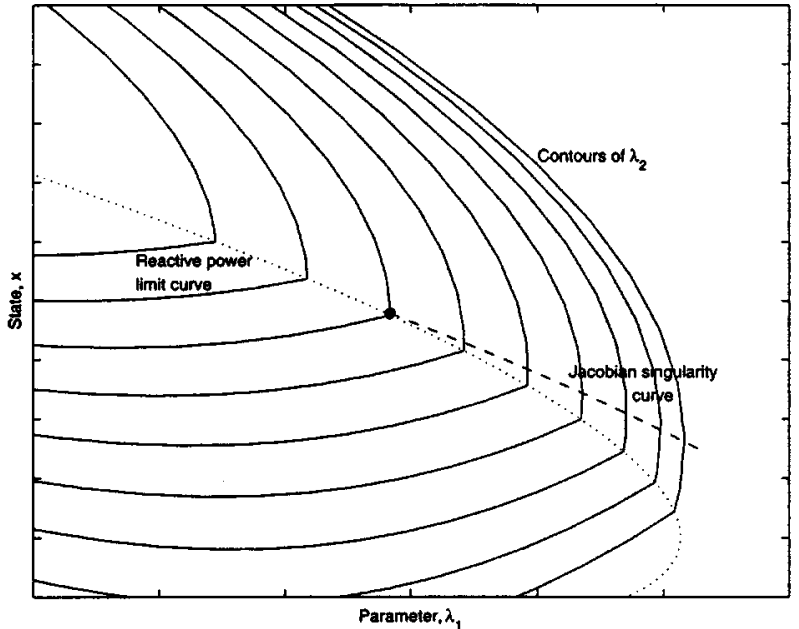

Fig. 14. Influence of reactive power limits.

the reactive power limit is first encountered. The true boundary is composed of the dashed line together with the section of the dotted line to the left of the point where the two curves intersect.

The reactive power limit curve, i.e., the dotted curve in Fig. 14, can be obtained from a normal continuation method. There are two free parameters, $\lambda_{1}$ and $\lambda_{2}$, so the total number of unknowns is $n+2$. Also, there are nominally $n$ power flow equations. However along the reactive power limit curve, a voltage constraint and a reactive power constraint are both enforced at the generator bus [19]. This adds an extra constraint, resulting is a problem of $n+1$ equations in $n+2$ unknowns. A 1-manifold (curve) is therefore defined.

\section{CONCLUSION}

A knowledge of the solution boundary of the power flow problem is important for determining the robustness of operating points, and for evaluating strategies for improving robustness. A method of exploring that solution boundary has been developed.

Curves of points which lie on the solution boundary can be found using a predictor-corrector approach. The curves result from freeing two parameters and enforcing the power flow constraints together with the extra constraint that the power flow Jacobian must be singular. An Euler homotopy method has been used for producing the curves. The algorithm has been illustrated using small examples. However the approach extends naturally to larger systems.

Examples have demonstrated some of the possible forms that the solution boundary can exhibit. It appears that quite complicated behavior is possible. This could have a significant influence on the formulation of algorithms for optimally improving system robustness. It remains to fully explore these issues.

In an operational environment, security monitoring often involves a comparison of measured (or state estimator) quantities with loadability constraints. These constraints frequently take the form of nomograms determined off-line. The proposed algorithm allows on-line generation of such nomograms, ensuring more accurate assessment of security and transmission availability. 


\section{REFERENCES}

[1] T. Van Cutsem and C. Vournas, Voltage Stability of Electric Power Systems: Kluwer Academic Publishers, 1998.

[2] C. L. DeMarco and A. R. Bergen, "A security measure for random load disturbances in nonlinear power system models," IEEE Trans. Circuits and Systems, vol. CAS-34, no. 12, pp. 1546-1557, Dec. 1987.

[3] C. J. Tavora and O. J. M. Smith, "Equilibrium analysis of power systems," IEEE Trans. Power Apparatus and Systems, vol. PAS-91, no. 3, pp. 1131-1137, 1972.

[4] J. Jarjis and F. D. Galiana, "Quantitative analysis of steady state stability in power systems," IEEE Trans. Power Apparatus and Systems, vol. PAS-100, no. 1, pp. 318-326, Jan. 1981.

[5] R. Seydel, "Numerical computation of branch points in nonlinear equations," Numer. Math., vol. 33, pp. 339-352, 1979.

[6] G. Moore and A. Spence, "The calculation of turning points on nonlinear equations," SIAM Journal of Numerical Analysis, vol. 18, pp. 567-576, Jan. 1980.

[7] F. L. Alvarado and T. H. Jung, "Direct detection of voltage collapse conditions," in Proceedings: Bulk Power System Voltage Phenomena-Voltage Stability and Security, Jan. 1989, EPRI Report EL-6183.

[8] I. Dobson, "Computing a closest bifurcation instability in multidimensional parameter space," Journal of Nonlinear Science, vol. 3, pp. 307-327, 1993.

[9] T. Van Cutsem, "A method to compute reactive power margins with respect to voltage collapse," IEEE Trans. Power Systems, vol. 6, no. 1, pp. 145-156, Feb. 1991.

[10] C. B. Garcia and W. I. Zangwill, Pathways to Solutions, Fixed Points and Equilibria. Englewood Cliffs, NJ: Prentice Hall, 1981.

[11] G. B. Price, "A generalized circle diagram approach for global analysis of transmission system performance," IEEE Trans. Power Apparatus and Systems, vol. PAS-103, no. 10, pp. 2881-2890, Oct. 1984.

[12] V. Ajjarapu and C. Christy, "The continuation power flow: A tool for steady state voltage stability analysis," IEEE Trans. Power Systems, vol. 7, no. 1, pp. 416-423, Feb. 1992.

[13] C. A. Cañizares and F. L. Alvarado, "Point of collapse and continuation methods for large AC/DC systems," IEEE Trans. Power Systems, vol. 8 , no. 1, pp. 1-8, Feb. 1993.
[14] H.-D. Chiang, A. J. Flueck, K. S. Shah, and N. Balu, "CPFLOW: A practical tool for tracing power system steady-state stationary behavior due to load and generation variations," IEEE Trans. Power Systems, vol. 10, no. 2, pp. 623-634, May 1995.

[15] R. Seydel, Practical Bifurcation and Stability Analysis, 2nd ed. New York: Springer-Verlag, 1994.

[16] A. Jepson and A. Spence, "Folds in solutions of two parameter systems and their calculation-Part I," SIAM Journal of Numerical Analysis, vol. 22, no. 2, pp. 347-368, Apr. 1985.

[17] W. C. Rheinboldt, "Computation of critical boundaries on equilibrium manifolds," SIAM Journal of Numerical Analysis, vol. 19, no. 3, pp. 653-669, June 1982.

[18] I. Dobson and L. Lu, "Voltage collapse precipitated by the immediate change in stability when generator reactive power limits are encountered," IEEE Trans. Circuits and Systems I, vol. 39, no. 9, pp. 762-766, Sept. 1992.

[19] I. A. Hiskens and B. B. Chakrabarti, "Direct calculation of reactive power limit points," International Journal of Electrical Power and Energy Systems, vol. 18, no. 2, pp. 121-129, 1996.

Ian A. Hiskens (S'77-M'80-SM'96) received the B.Eng. (Elec) and B.App.Sc. (Math) degrees from the Capricornia Institute of Advanced Education, Rockhampton, Australia in 1980 and 1983, respectively. He received the Ph.D. degree from the University of Newcastle, Australia in 1990. He was with the Queensland Electricity Supply Industry from 1980 to 1992, and was a Senior Lecturer at the University of Newcastle from 1992 to 1999 . He is currently a Visiting Associate Professor in the Department of Electrical and Computer Engineering at the University of Illinois at Urbana-Champaign

Robert J. Davy received the B.E. (Elec) and M.E. degrees from the University of Newcastle, Australia in 1993 and 1996, respectively. From 1996 to 1998, he was with Honeywell Ltd. He is currently a Data Analyst in the Wind Energy Research Unit within CSIRO Land and Water, Canberra, Australia. 